\title{
SANS Study of Understanding Mechanism of Cold Gelation of Globular Proteins
}

\author{
A. J. Chinchalikar ${ }^{1, *}$, Sugam Kumar ${ }^{1}$, V. K. Aswal ${ }^{1}$, J. Kohlbrecher ${ }^{2}$ and \\ A. G. Wagh ${ }^{1}$ \\ ${ }^{1}$ Solid State Physics Division, Bhabha Atomic Research Centre, Mumbai-400085, India \\ ${ }^{2}$ Laboratory of Neutron Scattering, Paul Scherrer Institut, CH - 5232 PSI Villigen, Switzerland \\ "E-mail:akshayc@barc.gov.in
}

\begin{abstract}
Small-angle neutron scattering (SANS) has been used to probe the evolution of interaction and the resultant structures in the cold gelation of globular proteins. The cold gelation involves two steps consisting of irreversible protein deformation by heating followed by some means (e.g. increasing ionic strength) to bring them together at room temperature. We have examined the role of different salts in cold gelation of preheated aqueous Bovine Serum Albumin (BSA) protein solutions. The interactions have been modeled by two Yukawa potential combining short-range attraction and long-range repulsion. We show that in step 1 (preheated temperature effect) the deformation of protein increases the magnitude of attractive interaction but not sufficient to induce gel. The attractive interaction is further enhanced in step 2 (salt effect) to result in gel formation. The salt effect is found to be strongly depending on the valency of the counterions. The gel structure has been characterized by the mass fractals.
\end{abstract}

Keywords: Protein gelation, Protein interaction, fractal structure, SANS

PACS: $61.05 . f g, 87.15 . \mathrm{km}, 64.60 . \mathrm{aq}$

\section{INTRODUCTION}

Gels can be defined as soft, deformable elastic solids consisting of a connected network of either particles or large molecules. In day-to-day life, gels appear in various forms such as soft, white, and curdy to translucent, rubbery, and firm. Globular proteins in aqueous solution denature when heated, leading in many cases to irreversible aggregation [1]. Above a critical protein concentration, proteins form gels with different structures and mechanical properties depending on the concentration, the amino acid sequence, $\mathrm{pH}$ and the ionic strength [2]. The solution environment provided at the time of gelation also decides the gel properties like rheological properties, water holding capacity etc.

The protein gelation in general involves heating up to gelation temperature where it may lose its native state functionality. On the other hand, the cold gelation is the novel method of producing protein gels at ambient temperatures which does not require high temperatures incompatible with the cell life [3]. In fact, this alternative way to obtain gels from proteins allows immediate applications in food technology, in biotechnology and, particularly, in tissue engineering where gel formation does not need high temperatures [4]. In contrast to heat induced gelation, the cold gelation is achieved through a two step process. The first step involves the heating treatment that causes the protein molecules to partially deform or unfold exposing reactive amino acids. In the second step the temperature is lowered (hence known as cold gelation) and then by means of salt, $\mathrm{pH}$ variation or pressure the gelation is induced $[5,6]$. Cold gelation has been studied most extensively for whey proteins but has been observed for other globular proteins as well [7].

In the present work, we report metal ion induced cold gelation of globular protein Bovine Serum Albumin (BSA) in aqueous solution at physiological $\mathrm{pH}$ 7. These metal ions can be viewed as micronutrients (necessary for the body) which get trapped into protein matrix thereby providing the ideal pathway for the manufacture of techno-functional food. The isoelectric point (pI) of BSA is 4.7 and possesses approximately 10 negative charges at the experimental $\mathrm{pH}$. The small-angle neutron scattering (SANS) has been utilized to probe the evolution of interactions and the resultant structures when system undergoes cold gelation. 


\section{EXPERIMENTAL}

Serum Albumin from Bovine (lyophilized) were obtained from Sigma-Aldrich and used as obtained. All the samples were prepared in $20 \mathrm{mM}$ phosphate buffer at $\mathrm{pH}$ 7.The $\mathrm{D}_{2} \mathrm{O}$ has been used as a solvent instead of $\mathrm{H}_{2} \mathrm{O}$ to provide better contrast in SANS experiments. The Small-angle neutron scattering measurements have been carried out at the SANS-I facility, Swiss Spallation Neutron Source SINQ, Paul Scherrer Institut, Switzerland. The wavelength of neutron beam used was $8 \AA$. The experiments were performed at sample-to-detector distances of 2 and $8 \mathrm{~m}$ to cover wave vector transfer $\mathrm{Q}(=4 \pi \sin \theta / \lambda$, where $2 \theta$ is the scattering angle and $\lambda$ is the wavelength of incident neutron) in the range of 0.005 to $0.3 \AA^{-1}$. The scattered neutrons were detected using twodimensional $96 \mathrm{~cm} \times 96 \mathrm{~cm}$ detector. The measurements have been carried out on pure $5 \mathrm{wt} \%$ BSA solutions with varying temperature $\left(30\right.$ to $\left.75^{\circ} \mathrm{C}\right)$ as well as on preheated $5 \mathrm{wt} \%$ BSA electrolyte solutions with varying salt concentration $(0-60 \mathrm{mM})$ and metal ion valency. The salts used are $\mathrm{NaCl}, \mathrm{CaCl}_{2}$ and $\mathrm{FeCl}_{3}$. Corrections were made for background and empty cell contributions and data were normalized to absolute cross-sectional unit using standard procedure.

\section{SANS ANALYSIS}

In SANS experiments, one measures the coherent differential scattering cross section per unit volume $(\mathrm{d} \Sigma / \mathrm{d} \Omega)$ as a function of $Q$ and is given by [8]

$$
\frac{d \Sigma}{d \Omega}(Q)=n\left(\rho_{p}-\rho_{s}\right)^{2} V_{p}^{2} P(Q) S(Q)+B
$$

where $\mathrm{n}$ is the number density of protein molecules, $\rho_{\mathrm{p}}$ and $\rho_{\mathrm{s}}$ are scattering length densities of macromolecule and solvent, respectively. $V_{\mathrm{p}}$ is the volume of the protein molecule. $P(Q)$ is the square of the form factor $F(Q)$ which contains information about shape and size of macromolecule. $S(Q)$ represents inter-particle structure factor and is determined by the interaction between protein molecules. $B$ is a constant term due to incoherent background which is mainly from the hydrogen content in the sample.

$P(Q)$ is decided by the shape and size of the particle. The $P(Q)$ for oblate ellipsoidal shape has been used for BSA protein molecule.

Interparticle structure factor $S(Q)$ depends upon the spatial arrangement of the particles and thereby gives the information about the inter-particle interaction. It may be approximated to unity if the sample is dilute and effectively there is no particle-particle interaction at average distances. The $S(Q)$ has been calculated using two Yukawa (2Y) potential under MSA closure.
The $2 \mathrm{Y}$ potential having 4 parameters $\left(K_{1}, K_{2}, Z_{1}\right.$ and $Z_{2}$ ) is expressed by [9]

$$
\begin{aligned}
\frac{V_{2 Y}(r)}{k_{B} T} & =-K_{1} \frac{\exp \left[-Z_{1}(r / \sigma-1)\right]}{r / \sigma}+K_{2} \frac{\exp \left[-Z_{2}(r / \sigma-1)\right]}{r / \sigma}(r>\sigma) \\
& =\infty(r<\sigma)
\end{aligned}
$$

and $\sigma\left[=2\left(a^{2} b\right)^{1 / 3}\right]$ is an effective hard sphere diameter of the protein macromolecule.

In the case of protein gelation as characterized by mass fractal structure, it is given by [10]

$$
S_{f}(Q)=1+\frac{1}{(Q R)^{D}} \frac{D \Gamma(D-1)}{\left[1+\frac{1}{(Q \xi)^{2}}\right]^{(D-1) / 2}} \sin \left\{(D-1) \times \tan ^{-1}(Q \xi)\right\}
$$

where $D$ is fractal dimension. $R$ and $\xi$ are minimum and maximum dimensions of fractal aggregates.

The scattering profiles as given by eq. (1) were smeared by the appropriate resolution function of the instrument to compare with the measured data. The parameters in the analysis were optimized by means of nonlinear least-square fitting program.

\section{RESULTS AND DISCUSSION}

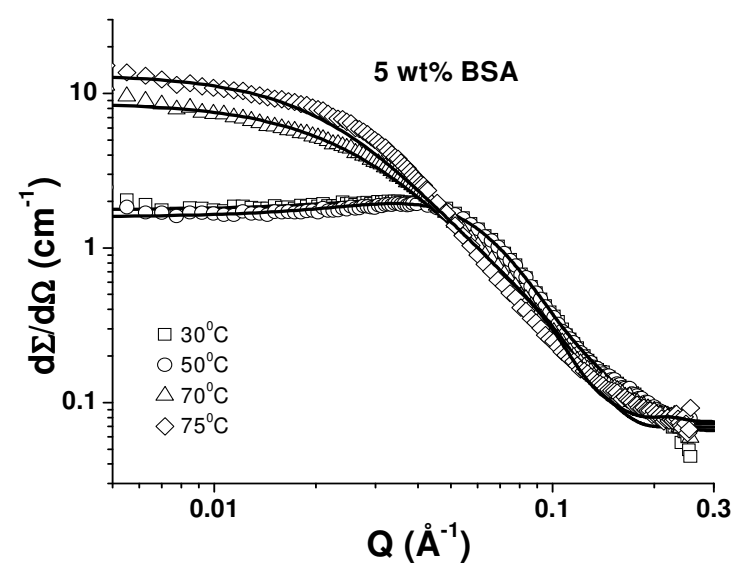

FIGURE 1. SANS data of 5 wt $\%$ aqueous BSA protein solution with varying temperature at $\mathrm{pH} 7$.

Fig. 1 shows the SANS data of 5 wt $\%$ BSA protein solution with variation in temperature at physiological $\mathrm{pH}$. The gelation temperature of BSA under present solution condition is $82^{\circ} \mathrm{C}$. The appearance of peak for SANS data $\left(30\right.$ and $\left.50^{\circ} \mathrm{C}\right)$ is due to correlation peak in their $S(Q)$ suggesting repulsive interaction between protein molecules. The data have been analyzed by using $2 \mathrm{Y}$ potential combining short-range attraction and long-range repulsion. The enhanced scattering in low $Q$ region with temperature $\left(>60^{\circ} \mathrm{C}\right)$ could be either due to the conformational changes or/and build-up of attractive interaction. Our analysis shows that there is an 
increase of about $30 \%$ in molecular volume of BSA along with many fold increase in attraction as accounted by $2 \mathrm{Y}$ potential. These observations may be understood in terms of enhanced dehydration due to an increase in temperature. However, no sign of gelation has been observed even up to highest temperature measured $\left(75^{\circ} \mathrm{C}\right)$.

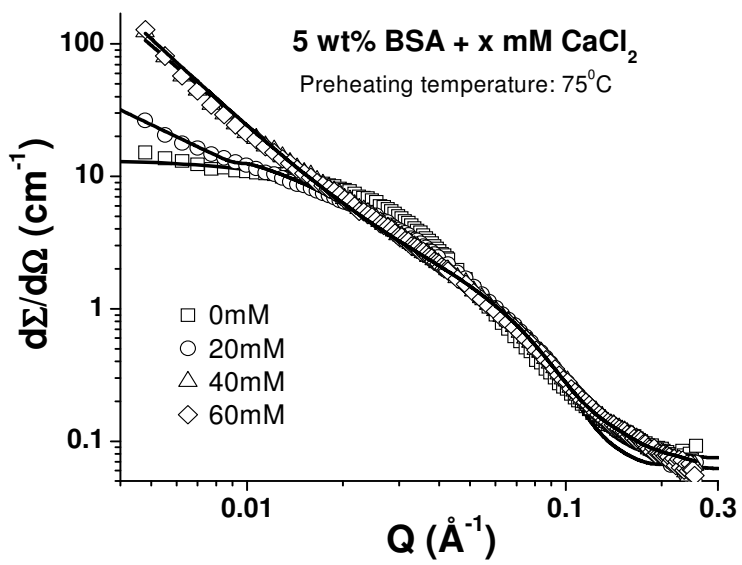

FIGURE 2. Room temperature $\left(30^{\circ} \mathrm{C}\right)$ SANS data of preheated $5 \mathrm{wt} \%$ BSA protein solution $(\mathrm{pH} 7)$ with variation in $\mathrm{CaCl}_{2}$ concentration. The preheating temperature was $75^{\circ} \mathrm{C}$.

The addition of salt in preheated protein solutions serves as the second step in cold gelation phenomenon. Fig. 2 presents the SANS data of BSA protein electrolyte solutions with varying salt $\left(\mathrm{CaCl}_{2}\right)$ concentration at room temperature $\left(30^{\circ} \mathrm{C}\right)$. All the sample solutions were heated at $75^{\circ} \mathrm{C}$ for two hours prior to an addition of salt. The data show scattering build-up in low $Q$ region with almost overlapping of data in high $Q$ region. The linear scattering build-up in low $Q$ region suggests the gel formation characterized by mass fractal structures. The fractal dimension increases with salt concentration finally saturating for concentrations more than $40 \mathrm{mM}$. It may be mentioned that throughout the analysis, the size of the protein molecules kept constant as obtained from the pure protein system at $75^{\circ} \mathrm{C}$.

The role of varying metal ion valency to induce gelation in protein solutions has also been investigated. We have chosen intermediate salt concentration $\left(20 \mathrm{mM} \mathrm{CaCl}_{2}\right)$ and the corresponding SANS data in presence of $\mathrm{NaCl}$ and $\mathrm{FeCl}_{3}$ (at same ionic strength) have been compared. The preheating temperature $\left(75^{\circ} \mathrm{C}\right)$ and time (2hrs) were kept same for all salts. The data show significant differences in low $Q$ region while the high $Q$ data remaining same. The linearity in low $Q$ region suggests the initiation of the gel formation. However, it is found that $60 \mathrm{mM} \mathrm{NaCl}$ is not sufficient for gel formation. The slope (measure of fractal dimension) is highest for $\mathrm{FeCl}_{3}$ (2.0) suggesting more closed packing of gel. The fact can be explained on the basis of effectiveness of multivalent metal salt ions to screen the charge and impose gelation. The higher the valency, lesser is the amount of salt required to achieve gelation.

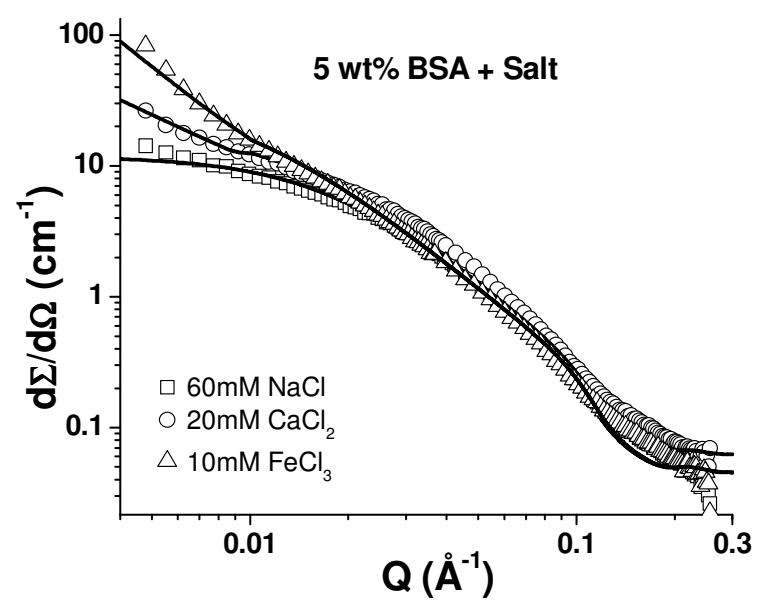

FIGURE 3. Comparison of SANS data (at $30^{\circ} \mathrm{C}$ ) of preheated $5 \mathrm{wt} \%$ BSA protein solution $(\mathrm{pH} 7)$ with variation in metal ion valency at same ionic strength.

To conclude, the temperature $\left(<\mathrm{T}_{\mathrm{Gel}}\right)$ enhances the attractive interaction with modifying the protein structures due to enhanced dehydration. Further, the addition of salt at room temperature is able to achieve cold gelation which strongly depends upon the metal salt concentration and its valency.

\section{REFERENCES}

1. W. S. Gosal and S. B. Ross-Murphy, Curr. Opin. Colloid. Interface Sci. 5, 188 (2000).

2. G. Baldini, S. Beretta and G. Chirico, Macromolecules 32, 6128 (1999).

3. K. Ako, T. Nicolai and D. Durand, Biomacromolecules, 11, 864 (2010).

4. G. Navarra, M. Leone and V. Militello, Biophys. Chem.131, 52 (2007).

5. G. Navarra, D. Giacomazza, M. Leone, F. Librizzi, V. Militello and P. L. San Biagio, Euro Biophys. J. 38, 437 (2009).

6. T. Phan-Xuan, D. Durand and T. Nicolai, Biomacromolecules 14, 1980 (2013).

7. C. M. Bryant and D. J. Mcclements, J. Food Sci. 65, 801 (2000).

8. J. S. Higgins and H. C. Benoit, Polymers and Neutron Scattering, Oxford: Clarendon Press, 1994.

9. A. J. Chinchalikar, V. K. Aswal, J. Kohlbrecher and A. G. Wagh, Phys. Rev. E 87, 062708 (2013).

10. J. Teixeira, J. Appl. Crystallogr. 21, 781 (1988). 